\title{
An Informatics Approach to Transformation Temperatures of NiTi-based Shape Memory Alloys
}

\author{
Dezhen Xue ${ }^{a, b}$, Deqing Xue ${ }^{a}$, Ruihao Yuan ${ }^{a}$, Yumei Zhou ${ }^{a}$, Prasanna V. Balachandran ${ }^{b}$, Xiangdong Ding ${ }^{\mathrm{a}}$, Jun Sun $^{\mathrm{a}}$, \\ Turab Lookman ${ }^{\text {b }}$ \\ ${ }^{a}$ State Key Laboratory for Mechanical Behavior of Materials, Xi'an Jiaotong University, Xi'an 710049, China \\ ${ }^{b}$ Theoretical Division, Los Alamos National Laboratory, Los Alamos, New Mexico 87545, USA
}

\begin{abstract}
The martensitic transformation serves as the basis for applications of shape memory alloys (SMAs). The ability to make rapid and accurate predictions of the transformation temperature of SMAs is therefore of much practical importance. In this study, we demonstrate that a statistical learning approach using three features or material descriptors related to the chemical bonding and atomic radii of the elements in the alloys, provides a means to predict transformation temperatures. Together with an adaptive design framework, we show that iteratively learning and improving the statistical model can accelerate the search for SMAs with targeted transformation temperatures. The possible mechanisms underlying the dependence of the transformation temperature on these features is discussed based on a Landau-type phenomenological model.
\end{abstract}

Keywords: Material informatics; Machine learning; Regression; Shape memory alloys; Transformation temperature

\section{Introduction}

Martensitic transformations are solid-to-solid displacive transitions characterized by a spontaneous strain accompanying a rapid change of crystal structure at the transformation temperature [1]. Shape-memory alloys (SMAs), undergoing reversible martensitic phase transformations, are widely used in applications ranging from sensors, actuators, eco-friendly refrigerators to energy conversion devices [2, 3, 4, 5, 6]. Most SMA based devices utilize either the temperature-induced or stressinduced martensitic transformation, so that the transformation temperature determines the temperature window for device use [7, 8, 9, 10] and is thus critical to applications of SMAs [1].

The transformation temperatures of SMAs are also strongly affected by their chemical composition [1, 8, 9. 10, 11, 12, 13]. As a result, the most common approach to tuning the transformation temperature for high or low temperature applications is to introduce elements to change the composition [14]. A number of studies

\footnotetext{
${ }^{*}$ Corresponding author

${ }^{* *}$ Corresponding author

Email addresses: xuedezhen@xjtu.edu.cn (Dezhen Xue), txl@lanl.gov (Turab Lookman)
}

Preprint submitted to Acta Materialia have focused on adding ternary elements to commercially used $\mathrm{Ti}_{50.0} \mathrm{Ni}_{50.0}$ to modify its transformation temperature and have identified specific elements that can elevate or lower the transformation temperature [14]. Alloying elements, such as $\mathrm{Fe}, \mathrm{Cr}, \mathrm{V}, \mathrm{Mn}$ and Co lower the transformation temperature [15, 16], whereas $\mathrm{Au}$, $\mathrm{Pd}, \mathrm{Pt}, \mathrm{Zr}$ and $\mathrm{Hf}$, increase the temperature [12, 14]. Moreover, the dependence of the martensitic transformation temperature on the concentration varies with the type of point defect. For example, in ternary alloys a 1.0 at\% of $\mathrm{Cr}$ replacing $\mathrm{Ni}$ in $\mathrm{Ti}_{50.0} \mathrm{Ni}_{50.0}$ lowers the transformation temperature by more than $150 \mathrm{~K}$, whereas for Co one needs more than 7.0 at\% to decrease the transformation temperature by $150 \mathrm{~K}[14,17]$.

However, in many cases ternary alloys are unable to satisfy the application requirements and so quaternary or even multi-component alloys need to be considered for desired properties [18, 19]. The increase in the number of elements is a serious impediment to developing SMAs with desired transformation temperatures as the chemical search space increases exponentially in size and complexity with alloying elements [19]. For $T i_{50.0} N i_{50.0}$ based alloys, the number of allowed candidate alloys can be as large as $10^{6}$ if three elements, whose concentrations are measured to $0.1 \%$, can replace $N i$. This is too vast a space to search experimentally. A 
method which allows us to effectively explore the complex search space to reduce time and costs is thus crucially important. From a practical point of view, desired are rapid and accurate predictions of the transformation temperatures based on different alloying elements and their concentrations.

Recently, there is growing interest in accelerating the materials discovery process of finding materials with targeted properties by reducing the number of costly and time-consuming trial and error experiments [19, [20, 21, 22, 23, 24, 25, 26]. To this end, databased statistical learning approaches are gradually making inroads into materials science. Some recent examples of informatics-driven design of new materials include predictions of molecular properties, transition states, structure classification of alloys, dielectric and piezoelectric properties and predictions of band gaps [19, 20, 21, 22, 23, 24, 25, 26].

In the present work, we explore a data-driven methodology that involves applying statistical learning tools to analyze correlations between transformation temperatures in SMAs and several scalar descriptors or features of electronic and crystal structure parameters in the constrained pseudo-quaternary system, $\mathrm{Ti}_{50}\left(\mathrm{Ni}_{50-x-y-z} \mathrm{Cu}_{\mathrm{x}} \mathrm{Fe}_{\mathrm{y}} \mathrm{Pd}_{\mathrm{z}}\right)$. We have previously used this system to identify $x, y, z$ in compounds with very low thermal hysteresis [19]. Our focus here is on developing a predictive data-driven capability to estimate the transformation temperature of unknown samples and to find alloys with targeted transformation temperatures in an accelerated manner. We find that a simple polynomial model, using three pre-selected material features or descriptors that capture chemical bonding and atomic size, works exceedingly well in describing the data. In this model, the transformation temperature depends linearly on the weighted average of the valence electron number (ven), Pauling electronegativity (en) and quadratically on Waber-Cromer's pseudopotential radius(dor) of the constituents of the alloys. Based on this relationship, we propose a simplified Landautype model to understand the mechanism controlling the transformation temperatures in NiTi-based alloys. Using the statistical learning model together with an adaptive design framework, we show that iteratively learning and improving the model accelerates the search for SMAs with targeted high transformation temperatures. In particular, we show how our design strategy predicts that $\mathrm{Ti}_{50} \mathrm{Ni}_{25} \mathrm{Pd}_{25}$ has the highest transition temperature of $189.56{ }^{\circ} \mathrm{C}$ in our restricted search space. Our experimental work validates this finding with a measured transformation temperature of $182.89^{\circ} \mathrm{C}$. Our methodology can be generalized to optimize other properties in multi-component SMAs.

Our paper is organized as follows. In Sec. 2 and 3 we introduce the experimental methods, our target property and dataset. Sec. 4 describes how we choose the most relevant features to make a prediction. Sec. 5 compares different regression models and identifies the best one. In Sec. 6 we show how the adaptive design framework utilizes the regression model to accelerate the search for alloys with targeted transformation temperatures. And finally in Sec. 77 we discuss the underlying physics of the dependence of the transformation temperature on the pre-selected features, which relate to the chemical bonding and atomic radii of the alloying elements.

\section{Experiments}

In the present study, we focus on the effects of chemical composition of NiTi-based alloys on the martensitic transformation temperature. We synthesized samples under identical conditions to avoid the effects of microstructural parameters such as grain size, precipitates and dislocations on the transformation temperature. The base ingot for the $\mathrm{Ti}_{50}\left(\mathrm{Ni}_{50-\mathrm{x}-\mathrm{y}-\mathrm{z}} \mathrm{Cu}_{\mathrm{x}} \mathrm{Fe}_{\mathrm{y}} \mathrm{Pd}_{\mathrm{z}}\right)$ alloy was made by arc melting from pure $99.9 \% \mathrm{Ti}$, 99.9\% $\mathrm{Ni}, 99.9 \% \mathrm{Cu}, 99.9 \% \mathrm{Fe}$ and $99.9 \% \mathrm{Pd}$ in an argon atmosphere. The ingot was hot-rolled into a 1.0 $\mathrm{mm}$ thick plate, the specimens for measurements were spark-cut from the plate and then solution-treated at $1273 \mathrm{~K}$ for $1 \mathrm{~h}$ in an evacuated quartz tube, followed by water quenching. Differential scanning calorimetry (DSC) measurements were made with a cooling/heating rate of $10 \mathrm{~K} \mathrm{~min}^{-1}$ to determine the martensitic transformation temperatures using exothermal/endothermic peaks.

\section{Dataset}

An input training data set is needed in order to build a quantitative statistical learning model for the NiTibased systems. Each material we are interested must be characterized or labelled via a representation in terms of one or more material descriptors or features, X. They represent aspects of structure, chemistry, bonding. Our machine learning method then learns a map or model connecting features $\mathrm{X}$ to a specific objective or material property $\mathrm{Y}$ (transformation temperature here), that is, $\mathrm{Y}=f(\mathrm{X})$. Thus, two main ingredients of the machine learning approach are the empirical model, $f$ and the features, $\mathrm{X}$. The learning algorithm to estimate $f$ must be trained and cross validated using a training dataset, which includes the measured targeted material property. 
The set of unexplored, possible materials whose targeted material properties have not been measured from the virtual dataset, on which $f$ will be applied to predict or estimate the targeted property.

As shown in Figure 1 we define an endothermic peak temperature, $T_{p}$, of the differential scanning calorimetry (DSC) curve as our target property for the transformation temperature. Other definitions such as $M_{s}$ and $M_{f}$, the start and finish temperatures for the martensitic transformation, and $A_{s}$ and $A_{f}$, the start and finish temperatures for the reverse transformation, could have also been adopted. We chose $T_{p}$ for simplicity and accuracy. We considered $\mathrm{Ti}_{50}\left(\mathrm{Ni}_{50-\mathrm{x}-\mathrm{y}-\mathrm{z}} \mathrm{Cu}_{\mathrm{x}} \mathrm{Fe}_{\mathrm{y}} \mathrm{Pd}_{\mathrm{z}}\right)$ alloys with $\mathrm{x}, \mathrm{y}$ and $\mathrm{z}$ compositions, which can vary in steps of $0.1 \%$ with constraints $50-x-y-z \geqslant 25 \%, x \leqslant 20 \%$, $\mathrm{y} \leqslant 7 \%$ and $\mathrm{z} \leqslant 25 \%$. This gives us $1,652,470$ possibilities in all. Of these, we synthesized 53 and measured their $T_{p}$ to form our training dataset, ensuring that the processing conditions were identical to minimize variability due to processing and microstructural effects. It is well known that changes in processing conditions affect microstructure (e.g. cast versus wrought gives rise to different microstructures in the case of the SMA Uranium Niobium). We tried to minimize this dependence on processing conditions so that the microstructural features would not vary as much across our samples and could be neglected as a first order effect. The remaining unexplored search space constitutes the virtual dataset, that is, $1,652,417$ potential alloys with unknown transformation temperatures.

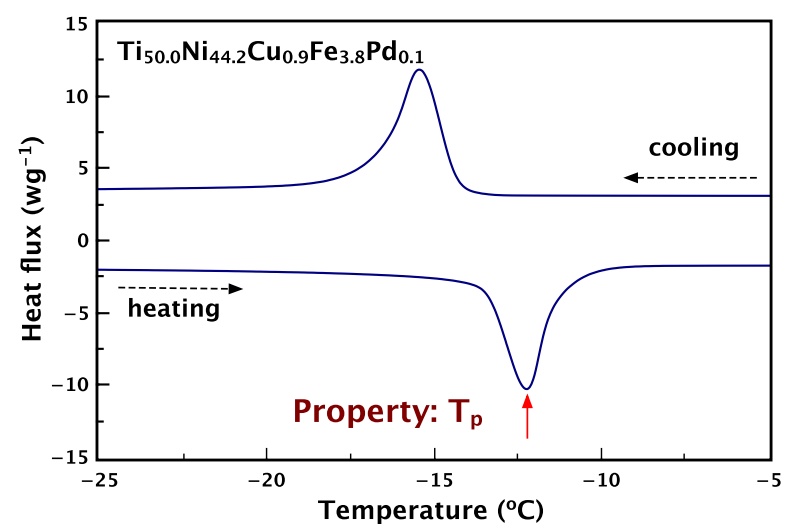

Figure 1: Definition of the property. The endothermic peak of the differential scanning calorimetry (DSC) curve, $T_{p}$, is our targeted property to optimize. The typical DSC result shown is from $\mathrm{Ti}_{50}\left(\mathrm{Ni}_{44.2} \mathrm{Cu}_{0.9} \mathrm{Fe}_{3.8} \mathrm{Pd}_{0.1}\right)$, one of our alloys in the training data.

\section{Feature Selection}

We discuss here how we choose the relevant features that form the input to the statistical learning model. A physical understanding of how the transformation temperature depends on composition has been the basis to intuitively screen the search space of SMA alloys and has been widely discussed in the literature. [8, 9, 10, 11, 13] For example, it is known that strong elastic softening, i.e., the decrease in elastic constant with temperature, occurs prior to the martensitic transformation and there is a critical value of the elastic constant at the transformation temperature [10]. Ren and Otsuka pointed out that the critical elastic constant is composition insensitive [11]. Therefore, the critical elastic constant can be reached at higher temperatures for softer materials and at lower temperatures for stiffer materials by tuning the composition. However, a direct mapping between the composition and the elastic properties is not available. Zarinejad and Liu subsequently associated the weighted average valence electron number (ven) with the elastic properties of SMAs and rationalized that the transformation temperature and ven are strongly correlated [8]. Frenzel et al. recently pointed out that the elastic constant is not sufficient by itself to predict the behavior of NiTi-based SMAs and suggested two factors controlling the transformation temperature: 1) a geometrical contribution as the result of changes in bonding by alloying elements; and 2) an intrinsic instability of the parent phase, which is related to the electronic structure [9].

Thus, the features we used comprised the set: Pauling electronegativity $(e n)$, metallic radius $(m r)$, valence electron number (ven), Waber-Cromer's pseudopotential radii (dor), Clementi's atomic radii (arc), and Pettifor chemical scale $(c s)$ [27, 28, 29, 30, 31, 32]. These features represent a coarse-grained analogue of electronic, bonding and radii properties in SMAs and provide a relatively simple physical basis for modeling structure-property relationship for the transformation temperature. Another consideration is that the material descriptors or features should be easily accessible for all possible materials, irrespective of the desired properties. The features, $\mathrm{X}$, for each $\mathrm{Ti}_{50}\left(\mathrm{Ni}_{50-\mathrm{x}-\mathrm{y}-\mathrm{z}} \mathrm{Cu}_{\mathrm{x}} \mathrm{Fe}_{\mathrm{y}} \mathrm{Pd}_{\mathrm{z}}\right)$ alloy in our composition space were uniquely described as a weighted fraction of the elemental features, that is,

$$
\mathrm{X}=f_{T i} \mathrm{X}^{T i}+f_{N i} \mathrm{X}^{N i}+f_{C u} \mathrm{X}^{C u}+f_{F e} \mathrm{X}^{F e}+f_{P d} \mathrm{X}^{P d},
$$

where $f_{T i}, f_{N i}, f_{C u}, f_{F e}$ and $f_{P d}$ are the atomic fractions of each element, $\mathrm{X}^{T i}, \mathrm{X}^{N i}, \mathrm{X}^{C u}, \mathrm{X}^{F e}$ and $\mathrm{X}^{P d}$ are the corresponding features of each element. Furthermore, Zarinejad and Liu also associated the transformation 
temperatures of SMAs with the average concentration of valence electrons which can be defined as the ratio of the number of valence electrons to the total number of electrons of the alloy (the atomic number) [8]. They observed a clear linear correlation between the transformation temperature and the average concentration of valence electrons [8]. Thus, we also define the ratio of each feature $X$ to the atomic number for each alloy to yield a new feature given by

$$
c \mathrm{X}=\frac{f_{T i} \mathrm{X}^{T i}+f_{N i} \mathrm{X}^{N i}+f_{C u} \mathrm{X}^{C u}+f_{F e} \mathrm{X}^{F e}+f_{P d} \mathrm{X}^{P d}}{f_{T i} Z^{T i}+f_{N i} Z^{N i}+f_{C u} Z^{C u}+f_{F e} Z^{F e}+f_{P d} Z^{P d}},
$$

where $Z^{T i}, Z^{N i}, Z^{C u}, Z^{F e}$ and $Z^{P d}$ are the atomic numbers of each element. Table 1 lists all the features we used in the present study.

Table 1: List of features used.

\begin{tabular}{cl}
\hline abbreviation & description \\
\hline numa & number of elements \\
arc & Pettifor chemical scale \\
en & Clementi's atomic radii \\
ven & Pauling electronegativity \\
mr & metallic radius \\
dor & Waber-Cromer's \\
& pseudopotential radii \\
anum & atomic number \\
mass & atomic mass \\
ccs & average concentration of $c s$ \\
carc & average concentration of arc \\
cmr & average concentration of $\mathrm{mr}$ \\
cen & average concentration of en \\
cven & average concentration of ven \\
cdor & average concentration of $\mathrm{dor}$ \\
cmass & average concentration of $\mathrm{mass}$
\end{tabular}

It is often the case that some or many of the features used in statistical learning models are not directly associated with the targeted property. Usually, including such features increases the dimensionality of the feature space and leads to unnecessary complexity in the resulting model. By removing these features, we are usually left with a simpler model, the results of which are then easier to interpret. Initially, features which are themselves highly correlated can be considered to contain essentially similar information. Therefore, we subject these features to a correlation filter to remove those that are uncorrelated to them. The Pearson correlation map is very useful for this purpose and is presented in Figure 2. It is calculated using the training data of 53 measurements with blue and red colors indicating posi- tive and negative correlations, respectively. The lighter the tone, the less significant is the corresponding correlation. In the upper- right half, the filled fraction of circles in each of the pie charts correspond to the absolute value of the associated Pearson correlation coefficient. Two clusters of data can be identified from the correlation map. In the right-bottom corner, ccs, carc, $\mathrm{cmr}$, cen, cven, cmass and cdor are highly correlated. Thus we choose $c c s$ to be typical of the information from this set of features. Another cluster present is that with $m r$, en, arc, anum, mass and dor. As they are all correlated, we selected $m r$, en and dor as representative of these features. Thus, after the correlation filter to down select features, we retain a subset of seven features that includes numa, cs, $m r$, en, ven, dor and ccs. Our preference for choosing $\mathrm{mr}$, en and dor was dictated by the fact that these features are more specific and physically meaningful in terms of their influence on SMA properties than just anum, mass and arc. The latter are rather general and some of them (e.g the radii) are essentially captured by other features in the set. Also, ven and $c s$ directly influence electronic properties whereas cmass and cdor are less specific in terms of their impact. Also, the clustering appears to reflect aspects of the training data primarily characterized by the different radii or electronic structure properties. However, the exact cause of this clustering is difficult to decipher from merely this type of analysis.

To further screen the features, we utilized the socalled subset selection method, which identifies a subset of the features that are mostly related to the targeted property [33]. If we have $p$ features, we fitted a separate least squares regression for each possible combination of those features. For example, we can fit $p$ models which contain only one feature, and can fit $p(p-1) / 2$ models which contain two features, and so forth. We then compared the performance of all the resulting models and identified the best one. The application of best subset selection to our problem is shown in Figure 3. Each plotted point corresponds to a least squares regression model fit using a different subset of the 7 features and the x-axis denotes the number of features used to fit the model. Figure 3 shows the mean squared error (MSE) and $R^{2}$ statistics for each model as a function of the number of features used to fit the model. The MSE captures the difference between the model predicted value, $\left(\hat{y}_{i}\right)$ and measured value $\left(y_{i}\right)$, and is given by,

$$
M S E^{\text {train }}=\frac{1}{n} \sum_{i=1}^{n}\left(y_{i}-\hat{y}_{i}\right)^{2}
$$




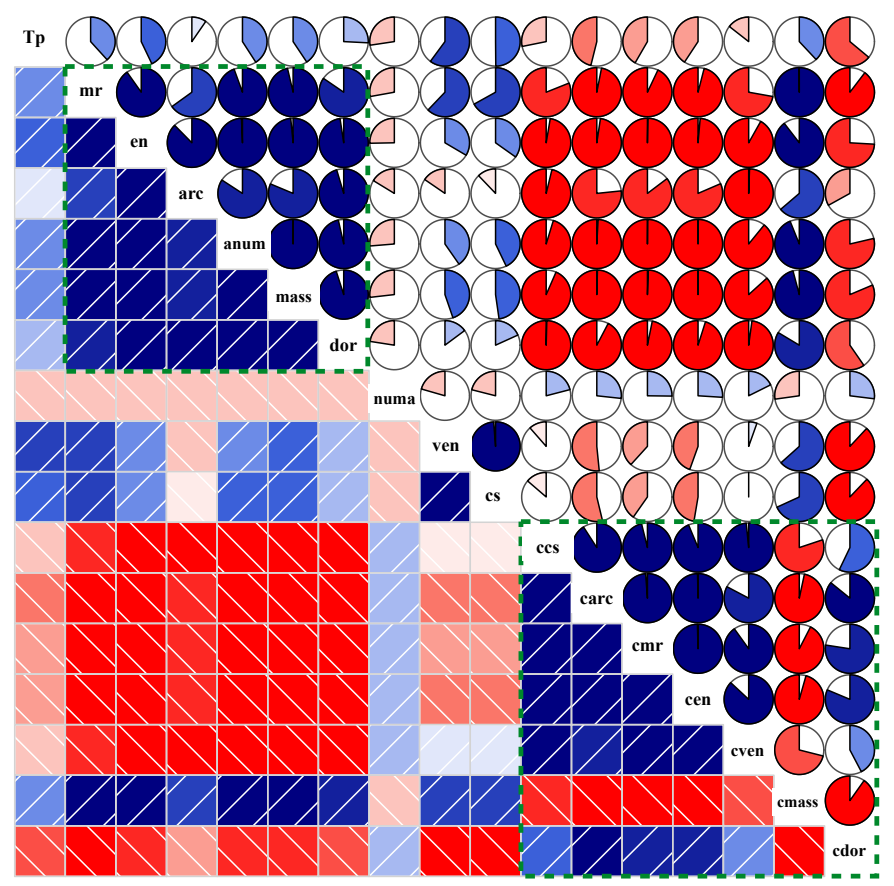

Figure 2: Pearson correlation map for features. A graphical representation of the Pearson correlation matrix for the initial features. Blue and red colors indicate positive and negative correlations, respectively. The lighter the tone used, the less significant is the corresponding correlation. The filled fraction of the circle in each of the pie charts corresponds to the absolute value of the associated Pearson correlations coefficient.

The smaller the $M S E^{\text {train }}$, the better we expect the model performance to be. The $R^{2}$ is an alternative measure of the performance and is given by

$$
R^{2}=1-\frac{\sum_{i=1}^{n}\left(y_{i}-\hat{y}_{i}\right)}{\sum_{i=1}^{n}\left(y_{i}-\bar{y}\right)}
$$

where $\bar{y}$ is the mean value of $y_{i}$. The $R^{2}$ value lies between 0 and 1, with 1 signifying excellent fits. In Figure 3, the red frontier lines connect the best models for each model size, according to $M S E^{\text {train }}$ and $R^{2}$ and we see that the two quantities improve as the number of features increases. However, there is little improvement in $M S E^{\text {train }}$ and $R^{2}$ beyond the use of 3 features and we limit ourselves to three in the subsequent statistical learning process. The best subset of 3 features includes $e n, v e n$ and $d o r$, and we use them to estimate the statistical learning models $f$ for $T_{p}=f(e n, v e n, d o r)$.

\section{Regression Models}

Our objective is to learn a quantitative relationship between a material property, (Y, transformation temperature) and the features characterizing the material
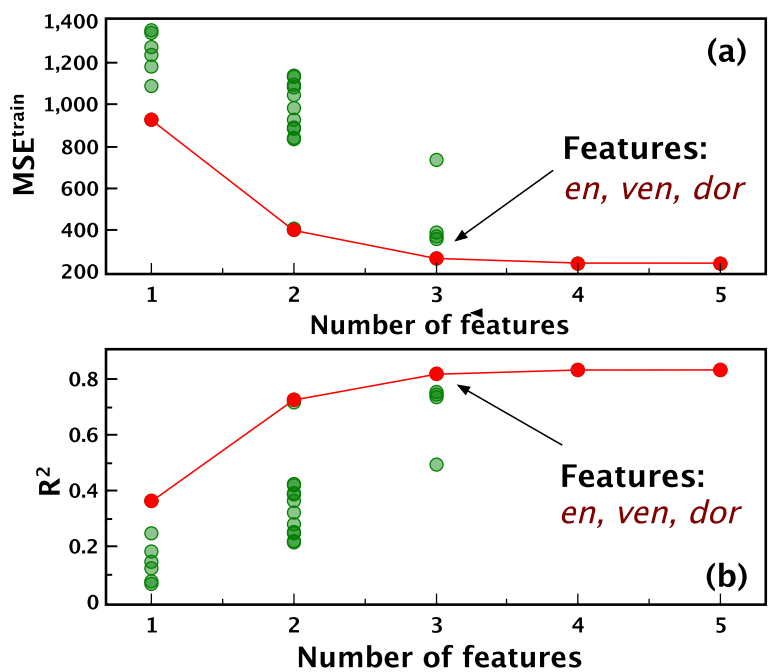

Figure 3: The mean squared error $\left(M S E^{\text {train }}\right)$ and $R^{2}$ of each possible linear model containing a subset of the seven features in our training data. The red frontier tracks the best model for a given number of features, according to $M S E^{\text {train }}$ and $R^{2}$. Both $M S E^{\text {train }}$ and $R^{2}$ show little improvement beyond 3 features. Thus, the subset of 3 features we employ includes en, ven and dor. 
$(\mathrm{X})$, i.e. $\mathrm{Y}=f(\mathrm{X})$. In general $f$ is unknown but statistical inference provides a means to estimate $f$. In the following, we estimate $f$ by using regression methods including linear (LIN) and polynomial regression (POLY), support vector regression with a linear kernel (SVR.lin), polynomial kernel (SVR.poly) and radial basis function kernel (SVR.rbf). These approaches identify how the response $T_{p}$ varies with en, ven, and $d o r$. As the set of features, en, ven, and dor are readily available, our learning approaches can yield accurate predictions for the targeted property, $T_{p}$, for all the unexplored data, as well as a way to understand how $T_{p}$ is affected by en, ven, and dor.

\subsection{Statistical learning models}

Estimating $f$ by linear regression (LIN) is a straightforward method for establishing a linear relationship between $\mathrm{X}$ and Y. For our problem, we can write this relationship as

$$
T_{p}=\beta_{0}+\beta_{1} \text { en }+\beta_{2} \text { dor }+\beta_{3} \text { ven },
$$

where $\beta_{i}$ are the coefficients for different features, which can be estimated by the method of least squares. The performance of the model can be evaluated by comparing $T_{p}$ measured and $T_{p}$ predicted for the data in the training set. In Figure $4(\mathrm{a})$, the predicted $T_{p}$ is plotted as a function of measured $T_{p}$. For a perfect model, the predicted $T_{p}$ will be exactly the same as the measured $T_{p}$ and all the data points will fall along the $45^{\circ}$ diagonal line in our plot. In practice, there will be variations which are desirable so that we do not overfit the data. Thus, the training error $\left(M S E^{\text {train }}\right)$, which can be calculated from Equation 3 , is used to evaluate the performance of our model on the training data.

In general, how well the model works on the training data is not crucial. Rather, we are interested in the accuracy of the predictions that we obtain when we apply our model to unexplored virtual data. There are various methods to estimate the test error, which can be used to select the best model. Resampling methods repeatedly draw samples from a training data set and refit a model of interest on each sample to obtain additional information about the fitted model, for example, information to evaluate the performance of the model on unseen or test data.

Bootstrap resampling is commonly used to evaluate how robust a model is by assigning measures of accuracy to sample estimates. It relies on sampling with replacement and we utilize it to quantify the uncertainty associated with prediction from the statistical learning on the training data, i.e., to obtain the mean $(\mu)$ and standard error $(\sigma)$ associated with the outcome of the model. Our training set contained $n=53$ observations, from which we randomly selected $n$ observations to produce a bootstrap data set. The sampling is performed with replacement, which means that the same observation can occur more than once in the bootstrap dataset. We trained a new LIN model on this bootstrap dataset, and made predictions for all the data points in the training dataset. We repeated this procedure 1000 times and obtained 1000 sets of predictions for the data in the training set for the mean $(\mu)$ and standard error $(\sigma)$. The bootstrap results for our LIN model are shown in the inset to Figure 4(a). The predicted $T_{p}$ of $\mathrm{y}$ axis is the $\mu$ and error bar is the $\sigma$. To estimate the test error, we replaced the predicted value $\hat{y}_{i}$ with the mean value $\mu_{i}$ in Equation 3 to define a MSE $E^{\text {boots }}$ given by,

$$
M S E^{\text {boots }}=\frac{1}{n} \sum_{i=1}^{n}\left(y_{i}-\mu_{i}\right)^{2} .
$$

Such $M S E^{\text {boots }}$ in some sense represents the test error of the model.

Cross-validation is used to assess the predictive capabilities of the model on unseen or test data. We typically hold-out a subset of the training observations from the fitting process and then apply the statistical learning model to the held-out observations. For our training set containing $n=53$ observations, we fit the LIN model on $n-1$ training observations, and the trained LIN model was used to predict $\hat{y}_{m}$ for the excluded observation (LOO, Leave One Out). Since the left out data point was not used in the fitting process, the squared error between this and the predicted value $\left(M S E=\left(y_{m}-\hat{y}_{m}\right)^{2}\right)$ provides an approximate unbiased estimate for the test error. Repeating this approach $n$ times on all the data points in turn, produces $n$ squared errors and CVerror, the average of those errors, is given by

$$
\text { CVerror }=\frac{1}{n} \sum_{m=1}^{n}\left(y_{m}-\hat{y}_{m}\right)^{2}
$$

In the following, we train several models and compute their $M S E^{\text {train }}, M S E^{\text {boots }}$, and CVerror.

\subsection{Model selection}

Polynomial regression extends the linear model to higher powers in the features (e.g. cubic regression includes $\mathrm{X}, \mathrm{X}^{2}$, and $\mathrm{X}^{3}$ ), and provides a simplified approach for non-linear fits to the data. To order $d$ in the features en, ven and dor, we have $T_{p}=\beta_{0}+\beta_{1}^{e n} e n+$ $\beta_{1}^{\text {dor }}$ dor $+\beta_{1}^{\text {ven }}$ ven $+\ldots+\beta_{d}^{\text {en }}$ en ${ }^{d}+\beta_{d}^{\text {dor }}$ dor $^{d}+\beta_{d}^{v e n}$ ven $^{d}$. The coefficients $\beta_{i}^{\text {en }}, \beta_{i}^{\text {ven }}$, and $\beta_{i}^{\text {dor }}$ can be easily estimated 

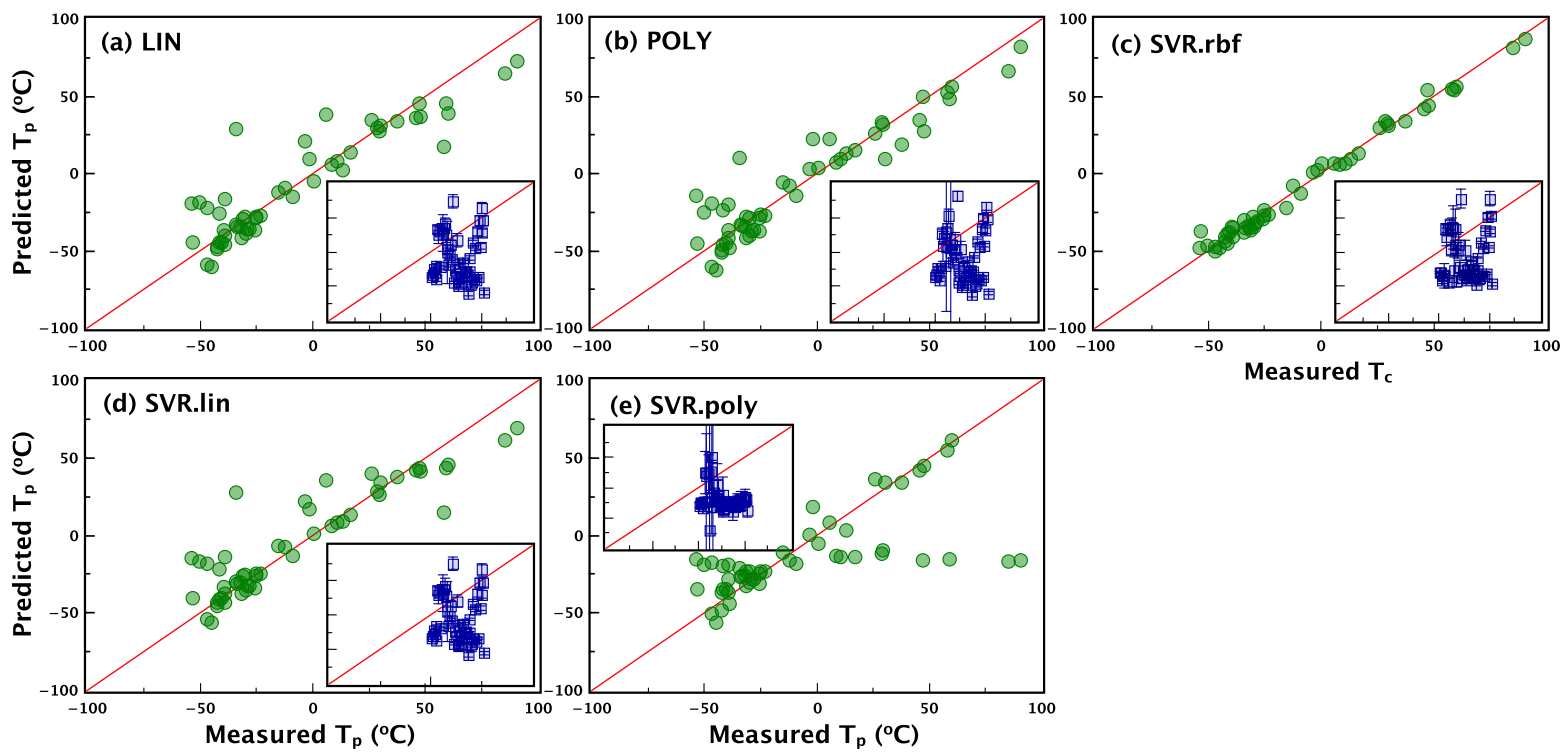

Measured $\mathrm{T}_{\mathrm{c}}$

Figure 4: The performance of the machine learning inference models on the training dataset. The predicted $T_{p}$ values from the inference model are plotted as a function of measured $T_{p}$ values. If the model is perfect, the predicted $T_{p}$ will be exactly the same as the measured $T_{p}$ and all the data points will align along the $45^{\circ}$ diagonal line. The insets plot the mean $(\mu)$ and standard deviation $(\sigma)$ of the predicted value from the inference model, obtained by bootstrap resampling from the initial 53 data points 1000 times. (a) linear regression (LIN); (b) polynomial regression (POLY); (c) Support vector regression with radial basis function kernel (SVR.rbf); (d) Support vector regression with linear kernel (SVR.lin); and (e) Support vector regression with polynomial kernel (SVR.poly). 


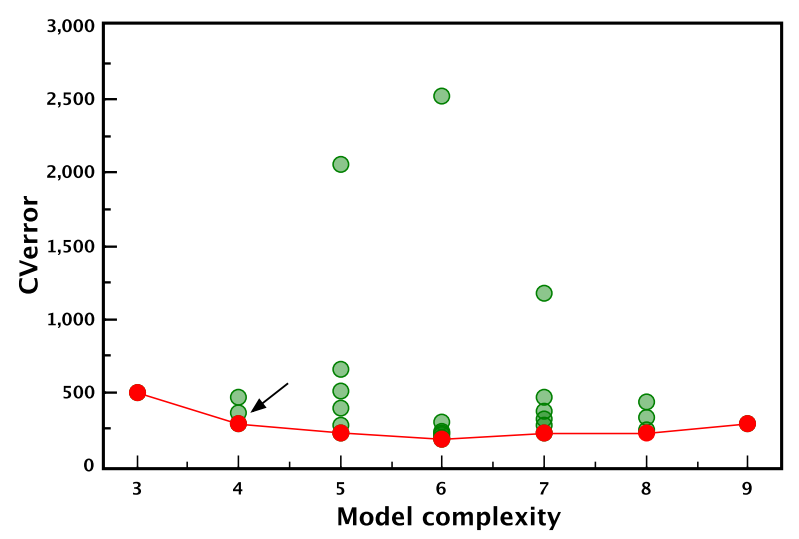

Figure 5: Cross validation error for different polynomial models (filled circles) using the three features en, ven and dor. The $\mathrm{x}$ axis is the sum of the orders of the terms in the polynomial models (e.g. the linear model has terms of order 1 for the three features, thus the sum is 3.) and is a measure of the model complexity. Small cross validation error indicates goodness of the model. The higher the sum of orders, the more complex is the polynomial model. The red front tracks the best polynomial model for a given sum. We chose the best model for the sum of 4 , to balance the trade-off between the goodness and model complexity.

using least squares regression. We tried different values of $d$ for en, dor and ven, respectively, and each combination of $d$ values for the three features corresponds to a polynomial model and determines its complexity. We used the cross-validation error, CVerror, to select the specific polynomial function for our targeted property, $T_{p}$. Figure 5 shows the cross-validation error of each polynomial model as a function of the model complexity in terms of the sum of orders $\left(\sum d_{i}, i=e n, d o r\right.$ and ven). The red curve connects the best polynomial models for given complexity and CVerror decreases as the order of the polynomial increases, revealing that the quality of the model improves. However, from the sum 6 onwards, there is little increase in CVerror. Given that we have a finite data size, we will clearly suffer from overfitting as the complexity increases. There is thus a trade-off between retaining model simplicity and overfitting. As CVerror does not decrease appreciably from values of 4 onwards, and certainly increases from 6 , we select the polynomial model corresponding to the sum of orders $=4$.

That is,

$$
T_{p}=\beta_{0}+\beta_{1}^{e n} e n+\beta_{1}^{d o r} d o r+\beta_{1}^{\text {ven }} \text { ven }+\beta_{2}^{\text {dor }} d \text { dor }^{2},
$$

which adds to the LIN model a quadratic term in dor. (This model has the lowest CVerror compared to the other possibilities with sum of orders $=4$.) Later we will show that the quadratic term is important in predicting the transformation temperature $T_{p}$. Figure 4 b) shows the predicted $T_{p}$ as a function of measured $T_{p}$, indicating a much improved model. The means and errors from bootstrapped samples in the inset to Figure 4(b) indicate that POLY is an improvement in terms of test error when compared to LIN.

Support vector regressors (SVRs) are kernel-based statistical learning approaches that are widely used in pattern recognition and imaging [34, 35]. They use an implicit mapping $\Phi$ of the input data into a highdimensional feature space defined by a kernel function, i.e., a function returning the inner product < $\Phi(x), \Phi\left(x^{\prime}\right)>$ between the images of two data points $x$, $x_{0}$ in the feature space. The learning then takes place in the feature space, and the data points only appear inside dot products with other points. We utilized SVR with a linear kernel (SVR.lin), radial basis function (SVR.rbf) kernel, and polynomial kernel (SVR.poly). The metaparameters for the SVR implementations were found using cross validation. Figure 4(c) - (e) show the performance of SVR.rbf, SVR.lin and SVR.poly models on our data. The SVR.rbf model performs very well on the data compared to SVR.lin or POLY; SVR.poly is the worst performer.

We computed the training and test errors for the above models, i.e., MSE ${ }^{\text {train }}$, MSE ${ }^{\text {boots }}$, and CVerror, using Equation 3, Equation 6, and Equation 7] Figure 6 (a) shows $M S E^{\text {train }}$ for five different models. It is clear the SVR.rbf out-performs all the other models, followed by POLY. Figure (b) and (c) show $M S E^{\text {boots }}$ and CVerror, estimates for the test errors with SVR.rbf outperforming all the other models with POLY next best. However, the outcome from SVR.rbf is not often easy to interpret and so to glean some understanding of the physics underlying the transformation temperature for SMAs, we will use the POLY model in the following.

\subsection{Validation}

Our training dataset is rather small and the estimates from $M S E^{\text {boots }}$ and CVerror of the test error for the training dataset may not accurately represent the full range of transformation temperatures. In addition, even though our machine learning models were built on the weighted atomic features, en, ven and dor for $\mathrm{Ti}_{50}\left(\mathrm{Ni}_{50-\mathrm{x}-\mathrm{y}-\mathrm{z}} \mathrm{Cu}_{\mathrm{x}} \mathrm{Fe}_{\mathrm{y}} \mathrm{Pd}_{\mathrm{z}}\right)$, they are not restricted to those particular alloying elements and we can apply them to predict transformation temperatures of other alloying elements.

We therefore collected 23 data points from Frenzel et al. [9], who published a high precision dataset on the transformation temperatures of NiTi-based SMAs, with 

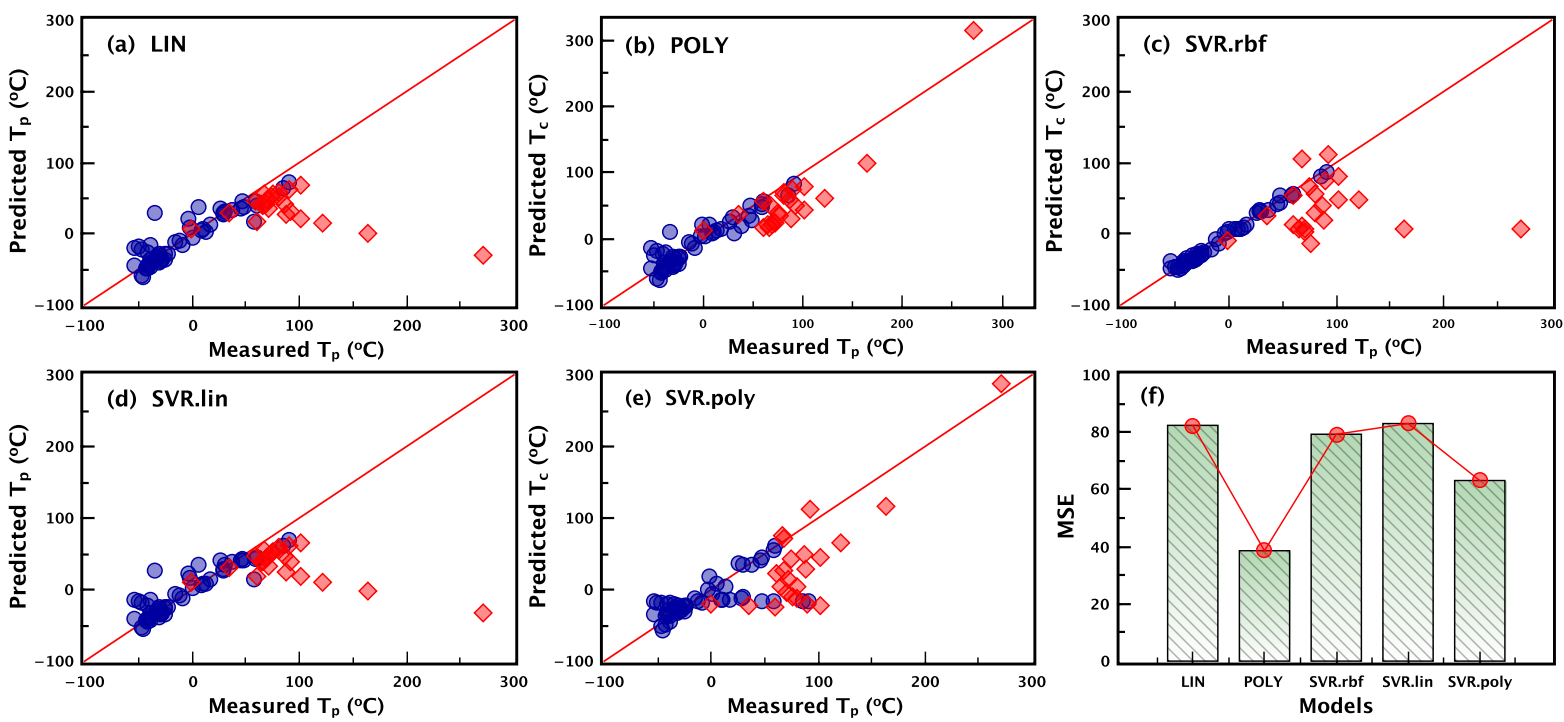

Figure 7: The performance of the machine learning inference models on our test dataset. (a) - (e) The predicted $T_{p}$ values from the inference model are plotted as a function of measured $T_{p}$ values. The red points are from our test dataset and the blue points are from our training dataset. (f) Comparison of different models in terms of the MSE on the test dataset, suggesting the POLY is the best performing model.

the constraint of $\mathrm{Ti} \%=50$ as we are focusing on replacing Ni in the present study. Figure 7)a)-(e) show how our model behaves on this 23 test data set. It appears that the LIN, POLY and SVR.lin perform fairly well for transformation temperatures below $100{ }^{\circ} \mathrm{C}$. SVR.rbf behaves well only for about 8 data points, even though it is the best performing model based on $M S E^{\text {boots }}$ and CVerror. Although SVR.poly predicts well the high temperature data points, it is the POLY model that is quite predictive for the whole temperature range. Figure 7 (f) shows the MSE error calculated from Equation 3 for the five models, indicating that the POLY model has the lowest error for the test data. The coefficients of the POLY model are shown in the first column of Table 2 Whether the same POLY model or one that is slightly different would apply to situations of varying Ti\% is difficult to predict. However, we expect the features to be essentially the same.

Although we have an adequate model, such as POLY, a key question is what is the optimal design strategy for finding SMA alloys with targeted transformation temperatures?

\section{Adaptive design}

We address this design problem by setting the goal of finding the highest transformation temperature alloy in our virtual dataset of unexplored alloys. We employed
Table 2: Coefficients of the POLY model on initial training dataset and updated training dataset.

\begin{tabular}{ccccc}
\hline & \multicolumn{2}{c}{ initial } & \multicolumn{2}{c}{ updated } \\
\cline { 2 - 5 } & Estimate & Error & Estimate & Error \\
\hline$\beta_{0}$ & -7.76 & 1.97 & -4.23 & 1.93 \\
$\beta_{1}^{\text {en }}$ & 1819.54 & 139.21 & 2738.32 & 207.39 \\
$\beta_{1}^{\text {dor }}$ & -1676.79 & 132.68 & -2495.48 & 202.57 \\
$\beta_{2}^{\text {dor }}$ & 64.31 & 14.61 & 141.49 & 14.89 \\
$\beta_{1}^{\text {ven }}$ & -184.36 & 30.53 & -184.59 & 30.21 \\
\hline
\end{tabular}

an adaptive design loop which uses a trade-off between exploration and exploitation of the results from our regressor model to guide the next experiment that needs to be performed [19]. The strategy we used is illustrated in Figure 8 and it can be exercised using the following steps.

1. Establish training and virtual datasets with features.

2. Learn an inference model for the relationship between features and the material property that we wish to optimize. It is important to consider uncertainties in the predicted properties.

3. Apply the trained inference model to the virtual dataset to predict property values with uncertainties of unexplored compunds.

4. Use a (selector) for design to suggest the next can- 

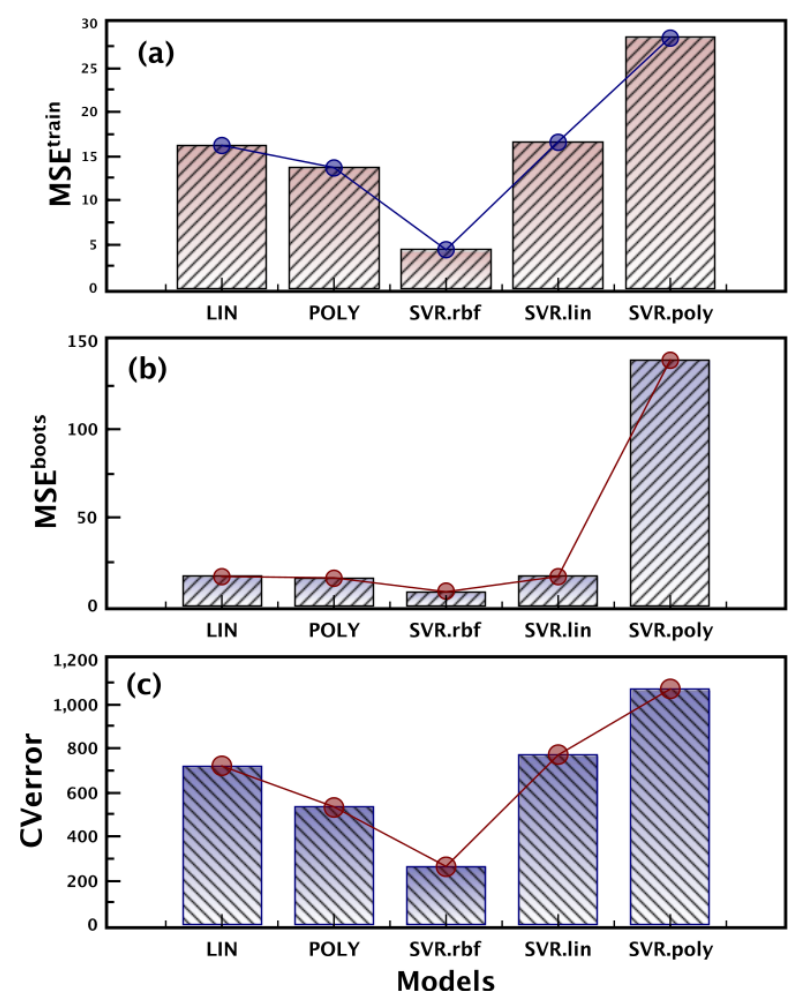

Figure 6: Performance of different models on our training data in terms of $\boldsymbol{M S E}^{\text {train }}, \boldsymbol{M S E}^{\text {boots }}$, and CVerror. (a) $M S E^{\text {train }}$, calculated using Equation 3 gives the training error for the inference model. (b) $M S E^{\text {boots }}$ and (c) CVerror, computed by Equation 6 and Equation 7 provide estimates of the test error. Form (a) to (c), SVR.rbf performs better than the others, POLY is next best.
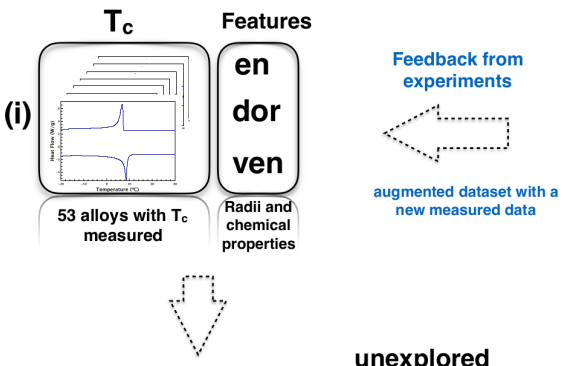

\section{Experiment}

(iv)

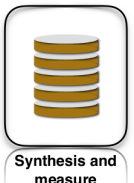

(ii)

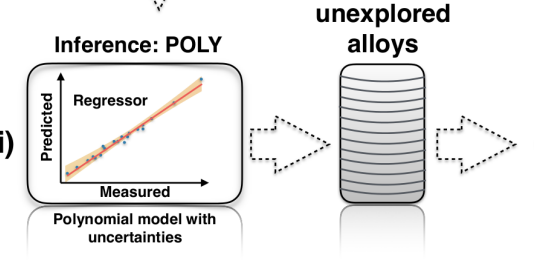

(iii)
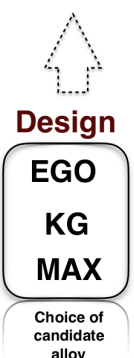

Figure 8: Adaptive design loop. (i) An initial experimental data set of 53 NiTi-based SMAs with known transformation temperature, and features serves as input to the inference model. (ii) The model is trained and cross-validated with the initial alloy data. The trained model in (ii) is applied to a data set of unexplored alloys, defined as our virtual dataset. (iii) The design chooses the best candidate for synthesis and characterization. (iv) The measured transformation temperature augments the initial data set to further improve the inference and design.

didate material for experiment by balancing the trade-off between exploitation (choosing the material with the best predicted property) and exploration (using the predicted uncertainties to study regions of search space where the model is less accurate).

5. Improve iteratively the inference model by feeding back the measurement results from experiments.

Steps 1 and Step 2 have already been discussed in Sections 2 to 5 , where POLY is determined to be the inference model of choice. To find the highest $T_{p}$ within our virtual dataset, we applied POLY to estimate $T_{p}$ for all $1,652,417$ potential alloys (Step3). The mean $(\mu)$ and standard deviation $(\sigma)$ of the predicted values were obtained by using bootstrap resampling, which considers representative random samples of some underlying distribution. The selectors choose or recommend the experiments which need to be performed next. In particular, the material to be synthesized next is selected to maximize the Expected Improvement, EI $(\mu, \sigma)$. Several selectors, all based on this concept, may be defined as follows:

- Max: $E I(\mu, \sigma)=\mu$ : Greedily chooses the material with maximum predicted value.

- EGO (efficient global optimization): Maximizes 
the "expected improvement" $E I(\mu, \sigma)=\sigma[\phi(z)+$ $z \Phi(z)]$, where $z=\left(\mu-\mu^{*}\right) / \sigma$ and $\mu^{*}$ is the maximum value observed so far in the training set. $\phi(z)$ and $\Phi(z)$ are the standard normal density and distribution functions, respectively.

- KG (knowledge gradient): $E I(\mu, \sigma)=\sigma[\phi(z)+$ $z \Phi(z)]$, where $z=\left(\mu-\mu^{* *}\right) / \sigma$, where $\mu^{* *}$ is the maximum value of either $\mu^{*}$ or $\mu^{\prime} ; \mu^{\prime}$ is the maximum predicted value in the virtual set.

We used these three selectors to choose the highest $T_{p}$ alloy from our virtual dataset (Step4). The results are shown in Table 3 The selector Max chose $\mathrm{Ti}_{50} \mathrm{Ni}_{49.9} \mathrm{Cu}_{0.1}$ as the best candidate, wheras selectors EGO and $\mathrm{KG}$ found that $\mathrm{Ti}_{50} \mathrm{Ni}_{25} \mathrm{Pd}_{25}$ has the highest expected improvement and is the alloy to be synthesized next. The selection of EGO and KG is physically meaninful as it is known that adding Pd will increase the transformation temperature. The $\mathrm{Ti}_{50} \mathrm{Ni}_{25} \mathrm{Pd}_{25}$ was then synthesized and its $T_{p}$ was determined to be $182.89^{\circ} \mathrm{C}$. Our POLY model predicted a value of $189.56^{\circ} \mathrm{C}$, which is in good agreement. The new compound was added to our training dataset, which then contained 54 data points (Step5) and the POLY model was subsequently retrained on the updated training dataset. The coefficients of the updated POLY model are shown in the "updated" column of Table 2

As seen in Table 3 the POLY model subsequently improved in terms of $M S E^{\text {train }}, M S E^{\text {boots }}$ and CVerror after it was retrained with the new data. The three selectors, Max, EGO and KG then all converged to the same selection $\mathrm{Ti}_{50} \mathrm{Ni}_{25} \mathrm{Pd}_{25}$, indicating no further room for improvement of $T_{p}$ within the virtual dataset. The predicted value from POLY model was $183.41{ }^{\circ} \mathrm{C}$, an improvement on the previous value. In summary, we executed two iterations of the adaptive design iterative loop and the convergence suggests high efficiency of the design loop. Thus the adaptive design framework here can be used to accelerate the search for SMAs with targeted transformation temperatures.

\section{Discussion and Summary}

As shown above, the POLY model reproduces the behavior of the transformation temperature in NiTi-based SMAs. It is then interesting to understand why the transformation temperature changes with the selected features and consequently with the alloying elements and concentration. We next consider a simple Landautype model to reveal the reasons behind the influence of these features on the martensitic transformation temperature.
In general, the alloying elements have different atomic sizes from the host alloy elements. Therefore, we expect the doped elements with different sizes to give rise to local lattice strains. Such local strains contribute to the total free energy of the system, and we can model the effects of induced strain by alloying elements on the transformation temperature in terms of a Landau model. The one dimensional Landau-free energy for shape memory alloys can be written in the form [36]

$$
F(T, \epsilon)=\frac{1}{2} A\left(T-T_{p}\right) \epsilon^{2}+\frac{1}{4} B \epsilon^{4}+\frac{1}{6} C \epsilon^{6},
$$

where $A, B$ and $C$ are elastic moduli of the materials (the later two are higher order moduli), $T_{p}$ is the transformation temperature, and $\epsilon$ is the order parameter. When dopants replace the host atoms, a local strain $e_{d}$ will be induced and will contribute to the total free energy by

$$
F(T, \epsilon)=\frac{1}{2} A\left(T-T_{p}\right) \epsilon^{2}+\frac{1}{4} B \epsilon^{4}+\frac{1}{6} C \epsilon^{6}+\alpha e_{d}^{2}+\beta \epsilon e_{d},
$$

where $\alpha$ and $\beta$ are coefficients associated with local strain induced by doping. At equilibrium, $\frac{\partial F}{\partial e_{d}}=0$, which gives,

$$
e_{d}=-\frac{\beta}{2 \alpha} \epsilon
$$

As a result, the effective free energy is,

$$
F(T, \epsilon)=\frac{1}{2} A\left(T-T_{p}^{*}\right) \epsilon^{2}+\frac{1}{4} \epsilon^{4}+\frac{1}{6} \epsilon^{6},
$$

where the $T_{p}^{*}$ is the effective transformation temperature when doped with defects, and is given by

$$
T_{p}^{*}=T_{p}+\frac{\beta^{2}}{2 A \alpha} .
$$

From Equation 13 it is clear that $\frac{\beta^{2}}{2 A \alpha}$ is the transformation temperature change due to the dopant. Therefore, this change controlling the SMA transformation temperature is influenced by the modulus $A$ and the local strains due to the effects of the atomic radii of the doped and host elements represented by $\beta$ and $\alpha$, respectively.

According to our POLY model, the transformation temperature linearly depends on the Pauling electronegativity (en) and valence electron number (ven) but quadratically depends on the $d$-orbital radii (dor). We selected dor to represent the atomic radii and thus captures one aspect of the transformation temperature variation for different dopants. Moreover, the quadratic dependence of transformation temperature on dor suggests that atomic radii may have even a stronger influence on the change in transformation temperature. 
Table 3: Comparison of the performance of POLY model on initial training dataset and updated training dataset and the predictions from initial POLY model and updated POLY model.

\begin{tabular}{|c|c|c|c|c|c|c|c|c|c|c|}
\hline \multirow{2}{*}{ data } & \multicolumn{3}{|c|}{ Model: POLY } & \multicolumn{2}{|c|}{ Selector: MAX } & \multicolumn{2}{|c|}{ Selector: EGO } & \multicolumn{2}{|c|}{ Selector: KG } & \multirow{2}{*}{$\begin{array}{l}\text { Prediction } \\
\mathrm{Ti}_{50} \mathrm{Ni}_{25} \mathrm{Pd}_{25}\end{array}$} \\
\hline & mse & mse & cv.error & alloy & mean & alloy & EI & alloy & EI & \\
\hline initial & 13.7 & 15.7 & 356.2 & $\mathrm{Ti}_{50} \mathrm{Ni}_{49.9} \mathrm{Cu}_{0.1}$ & 81.2 & $\mathrm{Ti}_{50} \mathrm{Ni}_{25} \mathrm{Pd}_{25}$ & 258.5 & $\mathrm{Ti}_{50} \mathrm{Ni}_{25} \mathrm{Pd}_{25}$ & 258.5 & 189.56 \\
\hline updated & 13.5 & 15.0 & 224.0 & $\mathrm{Ti}_{50} \mathrm{Ni}_{25} \mathrm{Pd}_{25}$ & 125.5 & $\mathrm{Ti}_{50} \mathrm{Ni}_{25} \mathrm{Pd}_{25}$ & 113.5 & $\mathrm{Ti}_{50} \mathrm{Ni}_{25} \mathrm{Pd}_{25}$ & 113.5 & 183.41 \\
\hline
\end{tabular}

In our choice of features, the en and ven capture the chemical bonding properties. It is known that the strong bonding gives rise to large resistance to shape/volume change, and consequently results in high bulk and shear moduli. As the elastic modulus softens and reaches a constant critical value before transformation upon cooling, the elastic modulus of the parent phase influences the transformation temperature. If the elastic modulus of the parent phase is large, cooling should continue to lower temperatures before the critical point is reached; hence the transformation temperature is decreased. On the contrary, if the alloying elements lower the elastic modulus of the parent phase, the critical point can be reached at higher temperatures and the transformation temperature should increase. Thus, the introduction of alloying elements in NiTi is accompanied by a change in the elastic modulus as the interatomic metallic bonding is affected, which is captured by the features en and ven.

Various chemical and microstructural factors can affect the transformation temperature of SMAs, however, in the present study, we consider only compositional changes of the alloy. Other factors, including aging, precipitation, point defects, dislocations and degree of order, which are also critical in determining the transformation temperature, are closely related with processing conditions. We expect that the same machine learning strategy we have discussed here can be utilized to model the influence of processing conditions, to understand how they determine the transformation and how to control the transformation temperature with them.

In summary, we have demonstrated a systematic learning and adaptive design framework to guide the synthesis and discovery of new alloys with targeted transformation temperatures. The same strategy can be used to discover new materials with other desired properties.

\section{Acknowledgement}

The authors gratefully acknowledge the support of National Basic Research Program of China (Grant No. 2012CB619401), the National Natural Science
Foundation of China (Grant Nos. 51571156, 51321003, 51302209, 51431007, and 51320105014), and Program for Changing Scholars and Innovative Research Team in University (IRT13034). We thank Prof. Xiaobing Ren for stimulating discussions. D.Z.X., P.V.B. and T. L. are grateful to the Laboratory Directed Research and Development (LDRD) program at Los Alamos National Laboratory (project \#20140013DR) for support.

\section{References}

[1] K. Otsuka, C. Wayman, Shape Memory Materials, Cambridge University Press, 1999. URL: https://books.google.com/ books?id=DvItE9XU1N8C

[2] K. Otsuka, T. Kakeshita, Science and technology of shapememory alloys: new developments, Mrs Bulletin 27 (2002) 91-100.

[3] J. San Juan, M. L. Nó, C. A. Schuh, Nanoscale shape-memory alloys for ultrahigh mechanical damping, Nature Nanotechnology 4 (2009) 415-419.

[4] J. Liu, T. Gottschall, K. P. Skokov, J. D. Moore, O. Gutfleisch, Giant magnetocaloric effect driven by structural transitions, Nature materials 11 (2012) 620-626.

[5] Y. Song, X. Chen, V. Dabade, T. W. Shield, R. D. James, Enhanced reversibility and unusual microstructure of a phasetransforming material, Nature 502 (2013) 85-88.

[6] C. Chluba, W. Ge, R. L. de Miranda, J. Strobel, L. Kienle, E. Quandt, M. Wuttig, Ultralow-fatigue shape memory alloy films, Science 348 (2015) 1004-1007.

[7] J. Cui, Y. S. Chu, O. O. Famodu, Y. Furuya, J. Hattrick-Simpers, R. D. James, A. Ludwig, S. Thienhaus, M. Wuttig, Z. Zhang, et al., Combinatorial search of thermoelastic shape-memory alloys with extremely small hysteresis width, Nat Mater. 5 (2006) 286-290.

[8] M. Zarinejad, Y. Liu, Dependence of transformation temperatures of niti-based shape-memory alloys on the number and concentration of valence electrons, Advanced Functional Materials 18 (2008) 2789-2794.

[9] J. Frenzel, A. Wieczorek, I. Opahle, B. Maaß, R. Drautz, G. Eggeler, On the effect of alloy composition on martensite start temperatures and latent heats in ni-ti-based shape memory alloys, Acta Materialia 90 (2015) 213-231.

[10] K. Otsuka, X. B. Ren, Factors affecting the ms temperature and its control in shape-memory alloys, in: Materials Science Forum, volume 394, Trans Tech Publ, 2002, pp. 177-184.

[11] X. B. Ren, K. Otsuka, Why does the martensitic transformation temperature strongly depend on composition?, in: Materials science forum, volume 327, Trans Tech Publ, 2000, pp. 429432.

[12] J. Ma, I. Karaman, R. D. Noebe, High temperature shape memory alloys, International Materials Reviews 55 (2010) 257-315. 
[13] J. Frenzel, E. P. George, A. Dlouhy, C. Somsen, M.-X. Wagner, G. Eggeler, Influence of ni on martensitic phase transformations in niti shape memory alloys, Acta Materialia 58 (2010) 34443458.

[14] K. Otsuka, X. Ren, Physical metallurgy of ti-ni-based shape memory alloys, Progress in materials science 50 (2005) 511678.

[15] Y. Zhou, D. Xue, X. Ding, Y. Wang, J. Zhang, Z. Zhang, D. Wang, K. Otsuka, J. Sun, X. Ren, Strain glass in doped ti $50($ ni $50-x d x)(d=c o, c r, m n)$ alloys: Implication for the generality of strain glass in defect-containing ferroelastic systems, Acta Materialia 58 (2010) 5433-5442.

[16] Y. Zhou, D. Xue, X. Ding, K. Otsuka, J. Sun, X. Ren, High temperature strain glass transition in defect doped ti-pd martensitic alloys, physica status solidi (b) 251 (2014) 2027-2033.

[17] T. Honma, Types and mechanical characteristics of shape memory alloys, Shape Memory Alloys (1984) 61-115.

[18] R. Zarnetta, R. Takahashi, M. L. Young, A. Savan, Y. Furuya S. Thienhaus, B. Maa§, M. Rahim, J. Frenzel, H. Brunken, Y. S. Chu, V. Srivastava, R. D. James, I. Takeuchi, G. Eggeler, A. Ludwig, Identification of Quaternary Shape Memory Alloys with Near-Zero Thermal Hysteresis and Unprecedented Functional Stability, Advanced Functional Materials 20 (2010) 1917-1923.

[19] D. Xue, P. V. Balachandran, J. Hogden, J. Theiler, D. Xue, T. Lookman, Accelerated search for materials with targeted properties by adaptive design, Nature Communications 7 (2016) 11241.

[20] D. Xue, P. V. Balachandrana, R. Yuan, T. Hu, X. Qian, E. R Dougherty, T. Lookman, Accelerated search for batio3-based piezoelectrics with vertical morphotropic phase boundary using bayesian learning, Proceedings of the National Academy of Sciences (2016).

[21] T. Lookman, P. V. Balachandran, D. Xue, J. Hogden, J. Theiler, Statistical inference and adaptive design for materials discovery, Current Opinion in Solid State and Materials Science (2016).

[22] P. Raccuglia, K. C. Elbert, P. D. Adler, C. Falk, M. B. Wenny, A. Mollo, M. Zeller, S. A. Friedler, J. Schrier, A. J. Norquist, Machine-learning-assisted materials discovery using failed experiments, Nature 533 (2016) 73-76.

[23] S. R. Kalidindi, M. De Graef, Materials data science: current status and future outlook, Annual Review of Materials Research 45 (2015) 171-193.

[24] P. V. Balachandran, D. Xue, J. Theiler, J. Hogden, T. Lookman, Adaptive strategies for materials design using uncertainties, Scientific Reports 6 (2016) 19660.

[25] P. V. Balachandran, D. Xue, T. Lookman, Structure-curie temperature relationships in batio 3-based ferroelectric perovskites: Anomalous behavior of (ba, cd) tio 3 from dft, statistical inference, and experiments, Physical Review B 93 (2016) 144111.

[26] M. Fernandez, P. G. Boyd, T. D. Daff, M. Z. Aghaji, T. K. Woo, Rapid and accurate machine learning recognition of high performing metal organic frameworks for co 2 capture, The journal of physical chemistry letters 5 (2014) 3056-3060.

[27] L. Pauling, The Nature of the Chemical Bond. IV. The Energy of Single Bonds and the Relative Electronegativity of Atoms, Journal of the American Chemical Society 54 (1932) 3570-3582.

[28] J. T. Waber, D. T. Cromer, Orbital Radii of Atoms and Ions, The Journal of Chemical Physics 42 (1965) 4116-4123.

[29] D. Pettifor, Bonding and Structure in Molecules and Solids, Oxford, Oxford, 1995.

[30] N. N. Greenwood, A. Earnshaw, Chemistry of the Elements, Second Edition, Butterworth-Heinemann, 1997.

[31] E. Clementi, D. L. Raimondi, W. P. Reinhardt, Atomic Screening Constants from SCF Functions. II. Atoms with 37 to 86
Electrons, The Journal of Chemical Physics 47 (1967) 13001307.

[32] K. M. Rabe, J. C. Phillips, P. Villars, I. D. Brown, Global multinary structural chemistry of stable quasicrystals, high- $T_{C}$ ferroelectrics, and high- $T_{c}$ superconductors, Phys. Rev. B 45 (1992) 7650-7676.

[33] G. James, D. Witten, T. J. Hastie, An introduction to statistical learning: with applications in R, Springer texts in statistics, Springer, New York, 2013. URL: http://opac.inria.fr/ record=b1135511

[34] C. J. Burges, A tutorial on support vector machines for pattern recognition, Data Mining and Knowledge Discovery 2 (1998) 121-167.

[35] A. J. Smola, B. Schölkopf, A tutorial on support vector regression, Statistics and Computing 14 (2004) 199-222.

[36] G. Barsch, Landau Theory of the Displacive Phase Transformations in Gold-Cadmium and Titanium-Nickel Alloys, Materials Science Forum 327-328 (2000) 3570-3582. 